\title{
RANDOM INTERVAL HOMEOMORPHISMS
}

\author{
LLUÍS ALSEDÀ AND MICHAŁ MISIUREWICZ
}

\begin{abstract}
We investigate homeomorphisms of a compact interval, applied randomly. We consider this system as a skew product with the two-sided Bernoulli shift in the base. If on the open interval there is a metric in which almost all maps are contractions, then (with mild additional assumptions) there exists a global pullback attractor, which is a graph of a function from the base to the fiber. It is also a forward attractor. However, the value of this function depends only on the past, so when we take the one-sided shift in the base, it disappears. We illustrate those phenomena on an example, where there are two piecewise linear homeomorphisms, one moving points to the right and the other one to the left.
\end{abstract}

\section{INTRODUCTION}

In this paper we investigate the properties of the systems of randomly applied orientation preserving homeomorphisms of the compact interval $[0,1]$. Such a system can be considered as a skew product with a mixed topological-measure structure. In the base we do not need any topology (although sometimes we have it), but we assume that we have there an ergodic measure preserving transformation of a probability space. In the fiber, which is an interval, we have orientation preserving homeomorphisms, depending in a measurable way on the point in the base.

We are interested in the existence of almost global attractors which are graphs of measurable functions from the base to the fiber. When we speak of an attractor, we mean a set towards which almost all orbits converge, and the convergence is considered fiberwise (only in the direction of a fiber). This agrees with the philosophy saying that the phase space is really only the fiber space (here, the interval).

Those systems and their attractors can be looked upon from various points of view (random systems, Strange Nonchaotic Attractors, Iterated Function Systems, nonautonomous systems, etc.), see [1].

Our main result is a detailed description of the behavior of a certain one-parameter family of piecewise linear random homeomorphisms. However, we precede it with some general results, which can be applied to very general random systems of interval homeomorphisms.

Note that 0 and 1 are fixed points of all orientation preserving homeomorphisms of $[0,1]$, so the products of the base space with $\{0\}$ and with $\{1\}$ (we will refer to those sets as level 0 and level 1) are invariant for the skew product. if they are attracting in the sense of negative fiberwise Lyapunov exponent, one expects their

Date: December 12, 2012.

2010 Mathematics Subject Classification. Primary: 37C55, 37C70.

Key words and phrases. Skew product, random system, attractor.

The first author has been partially supported by the MEC grant numbers MTM2008-01486 and MTM2011-26995-C02-01. 
basins of attraction to have positive measure. We prove that this is the case in a general situation, under some mild additional conditions. Our proof uses the same ideas as the proof by Bonifant and Milnor [3] in the special case.

In [3] the cases of attracting levels 0 and 1 (when the fiber maps have negative Schwarzian derivative) and repelling levels 0 and 1 (when the fiber maps have positive Schwarzian derivative) were considered separately. Here we join them together and consider an invertible map in the base. The two opposite types of behavior are observed depending whether the time goes to $+\infty$ or to $-\infty$. The common boundary of the basins of attraction of the levels 0 and 1 as the time goes to $-\infty$ is a graph of a measurable function from the base to the interval, is a forward attractor (statement (c) of Theorem 3.2) and a pullback attractor (statement (d) of Theorem 3.2) for the system.

In this general theorem one needs an additional assumption that the maps in the fibers are kind of contractions almost everywhere. Proving it is crucial in the study of this problem. In [3] this is achieved by the assumptions on Schwarzian derivatives of the maps. In our one-parameter family of maps this requires a careful proof. In fact, the contraction we get is very weak (although really it may turn out to be exponential almost everywhere; this is unknown to us).

Finally, we compare the invertible and non-invertible cases. Although the attractor in the invertible case depends only on the past in the base, it vanishes when we forget about the past (more precisely, it becomes the whole space). We call it the mystery of the vanishing attractor. While we described it already in [1], the system considered here is a much better illustration of this paradox.

The paper is organized as follows. In Section 2 we generalize the theorem of Bonifant and Milnor. In Section 3 we consider a general system with an invertible map in the base and prove a general theorem about its properties. In Section 4 we prove additional properties of the skew product under the assumption that the system in the base is a Bernoulli shift. In Section 5 we define our family of piecewise linear homeomorphisms and prove its contraction properties. In Section 6 we investigate our family of systems from the point of view of invariant measures. In Section 7 we compare the invertible and noninvertible systems.

Let us conclude this section with an observation and some questions. In the theory of interval maps (not random) negative Schwarzian derivative often substitutes expansion (see, e.g., $[4,5]$ ). The same happens in [3], where positive Schwarzian derivative gives us a form of contraction. However, in our piecewise linear system we also get a kind of contraction. What is the source of it? Does it have anything to do with some property resembling negative Schwarzian derivative? Can it be observed in non-random, say unimodal, maps?

\section{BOUNDARIES OF BASINS OF ATTRACTION}

Let us start with a very general situation. Let $\Omega$ be some space (later there will be an invariant measure on it), $R: \Omega \rightarrow \Omega$ a map, $I=[0,1], G: \Omega \times I \rightarrow \Omega \times I$ a skew product: $G(\vartheta, x)=\left(R(\vartheta), g_{\vartheta}(x)\right)$, and let $\pi_{2}$ be the projection from $\Omega \times I$ to $I$. We assume that each $g_{\vartheta}$ is an orientation preserving homeomorphism of $I$ onto itself.

The question is: if the level 0 set $\Omega \times\{0\}$ is an attractor, what can we say about the boundary of the basin of attraction? It can be defined as follows. 
Let $\varphi_{n, m}(\vartheta)$ be the unique number such that

$$
G^{n}\left(\vartheta, \varphi_{n, m}(\vartheta)\right)=\left(R^{n}(\vartheta), \frac{1}{m}\right) .
$$

This defines the function $\varphi_{n, m}: \Omega \rightarrow I$.

profinm Remark 2.1. Clearly, $\inf _{n \geq N} \varphi_{n, m}(\vartheta)$ is increasing in $N$ and decreasing in $m$.

Then we define a function $\varphi: \Omega \rightarrow I$ by

$$
\varphi(\vartheta)=\lim _{m \rightarrow \infty} \lim _{N \rightarrow \infty} \inf _{n \geq N} \varphi_{n, m}(\vartheta) .
$$

By Remark 2.1 the limits above exist.

Now we study the map $\varphi$ defined above.

lem1 Lemma 2.2. If $x<\varphi(\vartheta)$ then

$$
\lim _{n \rightarrow \infty} \pi_{2}\left(G^{n}(\vartheta, x)\right)=0 .
$$

If $x>\varphi(\vartheta)$ then (2.2) does not hold.

Proof. Assume first that $x<\varphi(\vartheta)$. Then, by Remark 2.1,

$$
\forall_{m} \quad x<\lim _{N \rightarrow \infty} \inf _{n \geq N} \varphi_{n, m}(\vartheta),
$$

SO

$$
\forall_{m} \exists_{N} \quad x<\inf _{n \geq N} \varphi_{n, m}(\vartheta),
$$

SO

$$
\forall_{m} \exists_{N} \forall_{n \geq N} \quad x<\varphi_{n, m}(\vartheta) .
$$

Observe that for every $m$ the inequality $x<\varphi_{n, m}(\vartheta)$ is equivalent to

$$
\left.\pi_{2}\left(G^{n}(\vartheta, x)\right)\right)<\frac{1}{m}
$$

and (2.2) follows.

Assume now that $x>\varphi(\vartheta)$. Then, again by Remark 2.1,

$$
\exists_{m} \quad x>\lim _{N \rightarrow \infty} \inf _{n \geq N} \varphi_{n, m}(\vartheta),
$$

SO

$$
\exists_{m} \forall_{N} \quad x>\inf _{n \geq N} \varphi_{n, m}(\vartheta),
$$

SO

$$
\exists_{m} \forall_{N} \exists_{n \geq N} \quad x>\varphi_{n, m}(\vartheta) .
$$

Similarly as above, for every $m$ the inequality $x>\varphi_{n, m}(\vartheta)$ is equivalent to

$$
\left.\pi_{2}\left(G^{n}(\vartheta, x)\right)\right)>\frac{1}{m}
$$

so (2.2) does not hold.

invgr Remark 2.3. It is easy to see that if $(2.2)$ holds when $x<\varphi(\vartheta)$ and does not hold when $x>\varphi(\vartheta)$, then the graph of $\varphi$ is $G$-invariant. 
lem2 Lemma 2.4. For a given $\vartheta \in \Omega$ assume that there exists $\eta>0$ and $\lambda_{n}(n=$ $0,1,2, \ldots)$ such that

for every $n$ and $x \in(0, \eta)$, and

$$
g_{R^{n}(\vartheta)}(x) \leq \lambda_{n} x
$$

$$
\limsup _{n \rightarrow \infty} \frac{1}{n} \sum_{k=0}^{n-1} \log \lambda_{k}<0 .
$$

Then $\varphi(\vartheta)>0$.

Proof. Take $r$ such that

$$
-r \in\left(\limsup _{n \rightarrow \infty} \frac{1}{n} \sum_{k=0}^{n-1} \log \lambda_{k}, 0\right) .
$$

Then for sufficiently large $n$ we have

$$
\prod_{k=0}^{n-1} \lambda_{k}=\exp \left(n \cdot \frac{1}{n} \sum_{k=0}^{n-1} \log \lambda_{k}\right)<e^{-n r} .
$$

Thus, we get

$$
\lim _{n \rightarrow \infty} \prod_{k=0}^{n-1} \lambda_{k}=0
$$

so, in particular,

$$
\max _{n}\left\{\prod_{k=0}^{n-1} \lambda_{k}\right\}<\infty
$$

Take any

$$
x_{0} \in\left(0, \frac{\eta}{\max \left\{1, \max _{n}\left\{\prod_{k=0}^{n-1} \lambda_{k}\right\}\right\}}\right) .
$$

Then we get for all $n$

$$
\pi_{2}\left(G^{n}\left(\vartheta, x_{0}\right)\right) \leq \prod_{k=0}^{n-1} \lambda_{k} \cdot x_{0}<\eta,
$$

and by (2.3) we get (2.2) with $x$ replaced by $x_{0}$. By Lemma 2.2 we get $\varphi(\vartheta) \geq x_{0}>$ 0 .

Let us now assume additionally that $\Omega$ is equipped with an $R$-invariant ergodic probability measure $\mu$, the maps $g_{\vartheta}$ depend on $\vartheta$ in a measurable way and they are all differentiable at 0 . Let $\Lambda$ be the exponent at level 0 , that is,

$$
\Lambda=\int_{\Omega} g_{\vartheta}^{\prime}(0) d \mu(\vartheta) .
$$

By the Birkhoff Ergodic Theorem, for almost every $\vartheta$ we have

$$
\lim _{n \rightarrow \infty} \frac{1}{n} \sum_{k=0}^{n-1} \log g_{R^{k}(\vartheta)}^{\prime}(0)=\Lambda \text {. }
$$

basin Theorem 2.5. Assume that $\Lambda<0$ and that at least one of the following assumptions is satisfied: 
i1 (i) the set $\left\{g_{\vartheta}: \vartheta \in \Omega\right\}$ is finite,

(ii) all functions $g_{\vartheta}$ are concave,

(iii) all functions $g_{\vartheta}$ are twice differentiable and there exists a constant $C$ such that $g_{\vartheta}^{\prime \prime}(x) / g_{\vartheta}^{\prime}(x) \leq C$ for all $\vartheta, x$.

Then there exists a measurable function $\varphi: \Omega \rightarrow I$, positive almost everywhere, such that for every $\vartheta \in \Omega(2.2)$ holds if $x<\varphi(\vartheta)$ and does not hold if $x>\varphi(\vartheta)$.

Proof. The function $\varphi$ is defined by (2.1) and it has the desired properties by Lemmas 2.2 and 2.4, provided the assumptions of Lemma 2.4 are satisfied for almost every $\vartheta$. To show that they are satisfied, it is enough to prove that there exists $\eta>0$ such that for every $\vartheta$ and $x \in(0, \eta)$

$$
\frac{g_{\vartheta}(x)}{x}<e^{-\Lambda / 2} g_{\vartheta}^{\prime}(0)
$$

(remember that $e^{-\Lambda / 2}>1$ ). Indeed, then we can take in Lemma 2.4

$$
\lambda_{k}=e^{-\Lambda / 2} f_{R^{k}(\vartheta)}^{\prime}(0),
$$

and by (2.4) we get for almost every $\vartheta$

$$
\limsup _{n \rightarrow \infty} \frac{1}{n} \sum_{k=0}^{n-1} \log \lambda_{k} \leq \lim _{n \rightarrow \infty} \frac{1}{n} \sum_{k=0}^{n-1} \log g_{R^{k}(\vartheta)}^{\prime}(0)-\frac{\Lambda}{2}=\frac{\Lambda}{2}<0 .
$$

Assume first that (i) is satisfied. If $\left\{g_{\vartheta}: \vartheta \in \Omega\right\}=\left\{h_{1}, \ldots, h_{m}\right\}$, then for every $i$, by the definition of the derivative and since $h_{i}(0)=0$, there is $\eta_{i}>0$ such that for all $x \in\left(0, \eta_{i}\right)$ we have $h_{i}(x) / x<e^{-\Lambda / 2} h_{i}^{\prime}(0)$. Now we take $\eta=\min \left\{\eta_{1}, \ldots, \eta_{m}\right\}$ and then for every $x \in(0, \eta)(2.5)$ holds.

Assume now that (ii) is satisfied. Then for every $\vartheta$ and $x$ we have

$$
\frac{g_{\vartheta}(x)}{x} \leq g_{\vartheta}^{\prime}(0)<e^{-\Lambda / 2} g_{\vartheta}^{\prime}(0)
$$

and we are done.

Assume finally that (iii) is satisfied. Set

$$
\eta=\min \left\{1,-\frac{\Lambda}{2 C}\right\} .
$$

Suppose that there are some $\vartheta$ and $x \in(0, \eta)$ for which $(2.5)$ does not hold. Then, by the Mean Value Theorem, there is $y \in(0, x)$ such that $g_{\vartheta}^{\prime}(y) \geq e^{-\Lambda / 2} g_{\vartheta}^{\prime}(0)$, that is,

$$
\log g_{\vartheta}^{\prime}(y)-\log g_{\vartheta}^{\prime}(0) \geq-\frac{\Lambda}{2} .
$$

Then there is $z \in(0, y)$ such that

$$
\frac{g_{\vartheta}^{\prime \prime}(z)}{g_{\vartheta}^{\prime}(z)}=\left(\log g_{\vartheta}^{\prime}\right)^{\prime}(z) \geq-\frac{\Lambda}{2 y}>-\frac{\Lambda}{2 \eta} \geq C,
$$

a contradiction with the assumption (iii). This completes the proof. 


\section{Two DIRECTIONS OF TIME}

Let us consider a skew product similar to the one from the preceding section, under an additional assumption that the map in the base is invertible. Then we can investigate what happens when the time goes to $+\infty$ and what happens when it goes to $-\infty$. To be in agreement with the theory of Strange Nonchaotic Attractors, we will think of the phenomena from the preceding section as occurring as the time goes to $-\infty$. Thus, we need new notation.

As before $\Omega$ is a space with a probability measure $\mu$. Now, $S: \Omega \rightarrow \Omega$ is an invertible measurable map (with $S^{-1}$ also measurable), for which $\mu$ is invariant and ergodic. The map $F: \Omega \times I \rightarrow \Omega \times I$ is a skew product, given by $F(\vartheta, x)=\left(S(\vartheta), f_{\vartheta}(x)\right)$, and each $f_{\vartheta}$ is an orientation preserving homeomorphism of $I$ onto itself.

We assume that the maps $f_{\vartheta}$ are differentiable at 0 and 1 , and define

$$
\Lambda_{0}=\int_{\Omega} f_{\vartheta}^{\prime}(0) d \mu(\vartheta), \quad \Lambda_{1}=\int_{\Omega} f_{\vartheta}^{\prime}(1) d \mu(\vartheta) .
$$

If both $\Lambda_{0}$ and $\Lambda_{1}$ are positive, then as the time goes to $-\infty$, the levels 0 and 1 are attracting. In many cases we can use Theorem 2.5 to conclude that their basins of attraction are nontrivial. However, there is no guarantee that the boundaries of those basins coincide. For this we need some kind of contraction in the fibers as the time goes to $+\infty$. Since the fiber maps are homeomorphisms, we cannot get contractions on closed intervals $[0,1]$. However, sometimes there is a kind of contraction on the open intervals $(0,1)$. One example of such a situation is given in the paper [3]. There all maps $f_{\vartheta}$ have positive Schwarzian derivative. Later in our paper we give a completely different example with two piecewise linear maps. However, there is no standard method of proving forward contraction for homeomorphisms. Therefore in our general theorem that follows, we make it one of the assumptions. In particular, we will use the following terminology, independently whether $S$ is invertible or not.

esscontr Definition 3.1. The skew product $F: \Omega \times I \rightarrow \Omega \times I$ is essentially contracting if for almost all $\vartheta \in \Omega$ and all $x, y \in(0,1)$, the distance

$$
\left|\pi_{2}\left(F^{n}(\vartheta, x)\right)-\pi_{2}\left(F^{n}(\vartheta, y)\right)\right|
$$

goes to 0 as $n \rightarrow \infty$.

If $\psi: \omega \rightarrow I$ is a measurable function, then we define the measure $\mu_{\psi}$, concentrated on the graph of $\psi$, as the lifting of the measure $\mu$, that is,

$$
\mu_{\psi}(A)=\mu\{\vartheta \in \Omega:(\vartheta, \psi(\vartheta)) \in A\} .
$$

main

$\operatorname{mi1}$

$\operatorname{mi2}$

mi3

ma1

Theorem 3.2. For a skew product $F$ as above, assume that

(I) $\Lambda_{0}, \Lambda_{1}>0$,

(II) either the set $\left\{f_{\vartheta}: \vartheta \in \Omega\right\}$ is finite, or all $f_{\vartheta}$ are diffeomorphisms of class $C^{2}$ with $\left|f_{\vartheta}^{\prime \prime}\right| /\left(f_{\vartheta}^{\prime}\right)^{2}$ bounded uniformly in $\vartheta$ and $x$,

(III) $F$ is essentially contracting.

Then there exists a measurable function $\varphi: \Omega \rightarrow(0,1)$ with the following properties:

(a) for almost every $\vartheta \in \Omega$, if $x<\varphi(\vartheta)$ then

$$
\lim _{n \rightarrow \infty} \pi_{2}\left(F^{-n}(\vartheta, x)\right)=0
$$


and if $x>\varphi(\vartheta)$ then

$$
\lim _{n \rightarrow \infty} \pi_{2}\left(F^{-n}(\vartheta, x)\right)=1
$$

ma2 (b) the graph of $\varphi$ is $F$-invariant,

ma3 (c) for almost every $\vartheta \in \Omega$ and every $x \in(0,1)$,

$$
\lim _{n \rightarrow \infty}\left|\pi_{2}\left(F^{n}(\vartheta, x)\right)-\varphi\left(S^{n}(\vartheta)\right)\right|=0,
$$

ma4 (d) for almost every $\vartheta \in \Omega$ and for every compact set $A \subset(0,1)$ and $\varepsilon>0$ there exists $N$ such that for every $n \geq N$

$$
\pi_{2}\left(F^{n}\left(\left\{S^{-n}(\vartheta)\right\} \times A\right) \subset(\varphi(\vartheta)-\varepsilon, \varphi(\vartheta)+\varepsilon) .\right.
$$

(e) if $\Omega$ is a metric compact space and $F$ is continuous, then for almost every $\vartheta \in \Omega$ and every $x \in(0,1)$, the measures

$$
\frac{1}{n} \sum_{k=0}^{n-1} F_{*}^{k}\left(\delta_{(\vartheta, x)}\right)
$$

converge (as $n \rightarrow \infty)$ in the weak-* topology to the measure $\mu_{\varphi}$.

Proof. Let us start by proving that the assumptions of Theorem 2.5 are satisfied for $G=F^{-1}$. Clearly, the exponent for $G$ at level 0 is equal to $-\Lambda_{0}$, so it is negative. Then, if there are finitely many fiber maps for $F$, then there are finitely many fiber maps for $G$, so (i) of Theorem 2.5 is satisfied. If all $f_{\vartheta}$ are diffeomorphisms of class $C^{2}$ with $\left|f_{\vartheta}^{\prime \prime}\right| /\left(f_{\vartheta}^{\prime}\right)^{2}$ bounded uniformly in $\vartheta$ and $x$, then to show that (iii) of Theorem 2.5 is satisfied, we just use the formula

$$
\frac{\left(f^{-1}\right)^{\prime \prime}(x)}{\left(f^{-1}\right)^{\prime}(x)}=\frac{-f^{\prime \prime}\left(f^{-1}(x)\right)}{\left(f^{\prime}\left(f^{-1}(x)\right)\right)^{2}} .
$$

Thus, by Theorem 2.5, there exists a measurable function $\varphi: \Omega \rightarrow(0,1]$, such that for almost every $\vartheta \in \Omega$, if $x<\varphi(\vartheta)$ then (3.1) holds. Similarly, there exists a measurable function $\tilde{\varphi}: \Omega \rightarrow[0,1)$, such that for almost every $\vartheta \in \Omega$, if $x>\tilde{\varphi}(\vartheta)$ then (3.2) holds for $\varphi$ replaced by $\tilde{\varphi}$. Clearly, $\varphi \leq \tilde{\varphi}$, so both functions have values in $(0,1)$. By Remark 2.3, the graphs of both functions are $F$-invariant (in particular, (b) holds). This means that $\varphi\left(S^{n}(\vartheta)\right)=\pi_{2}\left(F^{n}(\vartheta, \varphi(\vartheta))\right.$. Thus, (c) follows from (III). Similarly, (c) holds with $\varphi$ replaced by $\tilde{\varphi}$.

In such a way we get that

$$
\lim _{n \rightarrow \infty}\left|\varphi\left(S^{n}(\vartheta)\right)-\tilde{\varphi}\left(S^{n}(\vartheta)\right)\right|=0
$$

for almost every $\vartheta$. We want to prove that $\varphi=\tilde{\varphi}$ almost everywhere. If this is not true, then there exists $\varepsilon>0$ and a set $A \subset \Omega$ of positive measure such that $|\varphi(\vartheta)-\tilde{\varphi}(\vartheta)|>\varepsilon$ for every $\vartheta \in A$. However, by ergodicity of $\mu$, the trajectory of almost every point of $\Omega$ passes through $A$ infinitely many times, so we get a contradiction with (3.4). Thus, $\varphi=\tilde{\varphi}$ almost everywhere, and this completes the proof of (a).

To prove $(\mathrm{d})$, observe that there is $\delta>0$ such that $A \subset(\delta, 1-\delta)$. Take $\vartheta$ for which (a) holds. Then there is $N$ such that if $n \geq N$ then $\pi_{2}\left(F^{n}(\vartheta, \max (\varphi(\vartheta-\varepsilon), 0))<\right.$ $\delta$ and $\pi_{2}\left(F^{n}(\vartheta, \min (\varphi(\vartheta+\varepsilon), 1))>1-\delta\right.$. Then (3.3) holds. 
To prove (e), take $\vartheta$ for which (c) holds and such that $(\vartheta, \varphi(\vartheta))$ is generic for $\mu_{\varphi}$. The set of such $\vartheta$ has full measure. If $x \in(0,1)$ then the distance between $F^{n}(\vartheta, x)$ and $F^{n}(\vartheta, \varphi(\vartheta))$ goes to 0 as $n \rightarrow \infty$, and therefore (e) holds.

Let us finish this section by proving a theorem on invariant measures. It holds whether $S$ (and therefore, $F$ ) is invertible or not. Its proof is basically taken from [2]. We assume in it that there is topology in $\Omega$ in which $\mu$ is a Borel measure.

Theorem 3.3. Assume that $F$ is an essentially contracting skew product as above. Then there is at most one ergodic probability measure invariant for $F$ that projects to $\mu$ under $\left(\pi_{2}\right)_{*}$ and such that the measure of $\Omega \times\{0,1\}$ is 0 .

Proof. If there are two such measures, say $\nu_{1}$ and $\nu_{2}$, there is $\vartheta \in \Omega$ and two points $x, y \in(0,1)$, such that $(\vartheta, x)$ is generic for $\nu_{1},(\vartheta, y)$ is generic for $\nu_{2}$, and

$$
\lim _{n \rightarrow \infty}\left|\pi_{2}\left(F^{n}(\vartheta, x)\right)-\pi_{2}\left(F^{n}(\vartheta, y)\right)\right|=0
$$

Then in the weak-* topology, the averages of the images of the Dirac delta measure at $(\vartheta, x)$ converge to $\nu_{1}$ and the averages of the images of the Dirac delta measure at $(\vartheta, y)$ converge to $\nu_{2}$, and by $(3.5)$ we get $\nu_{1}=\nu_{2}$.

\section{Bernoulli Shift in the BASE}

Let us assume now that $(S, \Omega, \mu)$ is a Bernoulli shift on a finite alphabet. We can consider a two-sided shift $(\sigma, \Sigma, \mu)$ or a one-sided shift $\left(\sigma_{+}, \Sigma_{+}, \mu_{+}\right)$. We will write the points of $\Sigma$ and $\Sigma_{+}$as $\underline{\omega}=\left(\omega_{n}\right)_{n=\infty}^{\infty}$ or $\underline{\omega}=\left(\omega_{n}\right)_{n=0}^{\infty}$ respectively. We will also assume that the maps $f_{\underline{\omega}}$ depend only on $\omega_{0}$ (so there are only finitely many of them). The interpretation is that we are choosing those maps randomly and independently each time.

There is a natural projection $P: \Sigma \rightarrow \Sigma_{+}$. It is a semiconjugacy and it sends the measure $\mu$ to $\mu_{+}$.

In this context, let us look closer at the definition of the function $\varphi$, given at the beginning of Section 2.

pastphi Lemma 4.1. If $\underline{\omega}=\left(\omega_{n}\right)_{n=-\infty}^{\infty}$, then $\varphi(\underline{\omega})$ depends only on $\omega_{n}$ with $n<0$.

Proof. In our case, we have

$$
\varphi_{n, m}(\underline{\omega})=\pi_{2}\left(F^{n}\left(\sigma^{-n}, 1 / m\right)\right),
$$

so it depends only on $\omega_{n}$ with $n<0$. Thus, the same is true for $\varphi(\underline{\omega})$.

Now we can look what what happens when we project the measure $\mu_{\varphi}$ to the one-sided system.

Theorem 4.2. There exists a probability measure $\nu$ on $(0,1)$ such that

$$
\left(P \times \operatorname{id}_{I}\right)_{*}\left(\mu_{\varphi}\right)=\mu_{+} \times \nu .
$$

Proof. We can write $\Sigma=\Sigma_{-} \times \Sigma_{+}$, with

$$
\underline{\omega}=\left(\omega_{n}\right)_{n=-\infty}^{\infty}=\left(\underline{\omega}_{-}, \underline{\omega}_{+}\right)=\left(\left(\omega_{n}\right)_{n=-\infty}^{-1},\left(\omega_{n}\right)_{n=0}^{\infty}\right),
$$

where $\underline{\omega}_{-} \in \Sigma_{-}$and $\underline{\omega}_{+} \in \Sigma_{+}$. By Lemma 4.1, there exists a measurable function $\varphi_{-}: \Sigma_{-} \rightarrow(0,1)$ such that

$$
\varphi(\underline{\omega})=\varphi_{-}\left(\underline{\omega}_{-}\right) .
$$


On $\Sigma_{-}$there is a product measure $\mu_{-}$such that $\mu=\mu_{-} \times \mu_{+}$. We can identify in a natural way $\Sigma \times I=\left(\Sigma_{-} \times \Sigma_{+}\right) \times I$ with $\Sigma_{+} \times\left(\Sigma_{-} \times I\right)$. Then, by (4.1), we have $\mu_{\varphi}=\left(\mu_{-}\right)_{\varphi_{-}} \times \mu_{+}$, where $\left(\mu_{-}\right)_{\varphi_{-}}$is the measure on $\Sigma_{-} \times I$ defined similarly as $\mu_{\varphi}$.

Let $\pi_{-}: \Sigma_{-} \times I \rightarrow I$ be the natural projection. Set $\nu=\left(\pi_{-}\right)_{*}\left(\left(\mu_{-}\right)_{\varphi_{-}}\right)$. With our identification, we have $P \times \mathrm{id}_{I}=\mathrm{id}_{\Sigma_{+}} \times \pi_{-}$. We get

$\left(P \times \operatorname{id}_{I}\right)_{*}\left(\mu_{\varphi}\right)=\left(\operatorname{id}_{\Sigma_{+}} \times \pi_{-}\right)_{*}\left(\mu_{+} \times\left(\mu_{-}\right)_{\varphi_{-}}\right)=\left(\operatorname{id}_{\Sigma_{+}}\right)_{*}\left(\mu_{+}\right) \times\left(\pi_{-}\right)_{*}\left(\left(\mu_{-}\right)_{\varphi_{-}}\right)=\mu_{+} \times \nu$.

\section{Piecewise linear homeOmorphisms}

Now we consider a one-parameter family of random homeomorphisms of an interval, for which we can prove that the theory from the preceding sections applies.

The situation will be as in the preceding section. The system in the base will be the Bernoulli shift with probabilities $(1 / 2,1 / 2)$. The corresponding interval homeomorphisms, $f_{0}, f_{1}: I \rightarrow I$ will be piecewise liner with two pieces. Additionally, their graphs will be symmetric with respect to $(1 / 2,1 / 2)$, that is, $f_{1}(x)=1-f_{0}(1-x)$. For each map the point at which it is not linear can be considered as a critical point. As always, the situation is simpler if there is only one critical value, and by the symmetry, this common critical value has to be $1 / 2$. Since our maps are orientation preserving homeomorphisms, we have $f_{0}(0)=f_{1}(0)=0$ and $f_{0}(1)=f_{1}(1)=1$.

These conditions determine a one-parameter family of pairs of maps

$$
\begin{aligned}
& f_{0}(x)= \begin{cases}a x & \text { if } 0 \leq x \leq 1-c, \\
1-b(1-x) & \text { if } 1-c \leq x \leq 1,\end{cases} \\
& f_{1}(x)= \begin{cases}b x & \text { if } 0 \leq x \leq c, \\
1-a(1-x) & \text { if } c \leq x \leq 1 .\end{cases}
\end{aligned}
$$

where $a=\frac{1}{2(1-c)}, b=\frac{1}{2 c}$, and $0<c<1 / 2$ (see Figure 1 ). Observe that the harmonic mean of the slopes $a$ and $b$ is 1 , and that $0<a<1<b$.

We will apply $f_{j}, j=0,1$, when the 0 -th coordinate of $\underline{\omega} \in \Sigma$ (or in $\Sigma_{+}$) is $j$. That is, we consider skew products $F: \Sigma \times I \rightarrow \Sigma \times I$ given by $F(\underline{\omega}, x)=\left(\sigma(\underline{\omega}), f_{\omega_{0}}(x)\right)$, where $\underline{\omega}=\left(\omega_{n}\right)_{n=-\infty}^{\infty}$, and $F_{+}: \Sigma_{+} \times I \rightarrow \Sigma_{+} \times I$ given by $F_{+}(\underline{\omega}, x)=\left(\sigma_{+}(\underline{\omega}), f_{\omega_{0}}(x)\right)$, where $\underline{\omega}=\left(\omega_{n}\right)_{n=0}^{\infty}$.

We want to apply Theorem 3.2. Therefore we need to check that its assumptions are satisfied by $F$. Assumption (I) is satisfied because $a b=\frac{1}{4 c(1-c)}>1$. Assumption (II) is satisfied because there are only 2 maps $f_{\vartheta}$. Thus, we have to prove that $F$ is essentially contracting. As we mentioned earlier, this is a nontrivial thing to do.

The main idea is to find a homeomorphism from $(0,1)$ to $\mathbb{R}$ such that in the new metric in $(0,1)$, which we get by transporting back the natural metric from $\mathbb{R}$, both maps $f_{0}$ and $f_{1}$ are contractions. In fact, they will be very weak contractions (on the most of the space they will be isometries), so we need more work in order to prove that $F$ is essentially contracting.

Let $h:(0,1) \rightarrow \mathbb{R}$ be a homeomorphism given by the formula

$$
h(x)= \begin{cases}\log x-\log \frac{1}{2} & \text { if } x \leq \frac{1}{2} \\ \log \frac{1}{2}-\log (1-x) & \text { if } x>\frac{1}{2}\end{cases}
$$




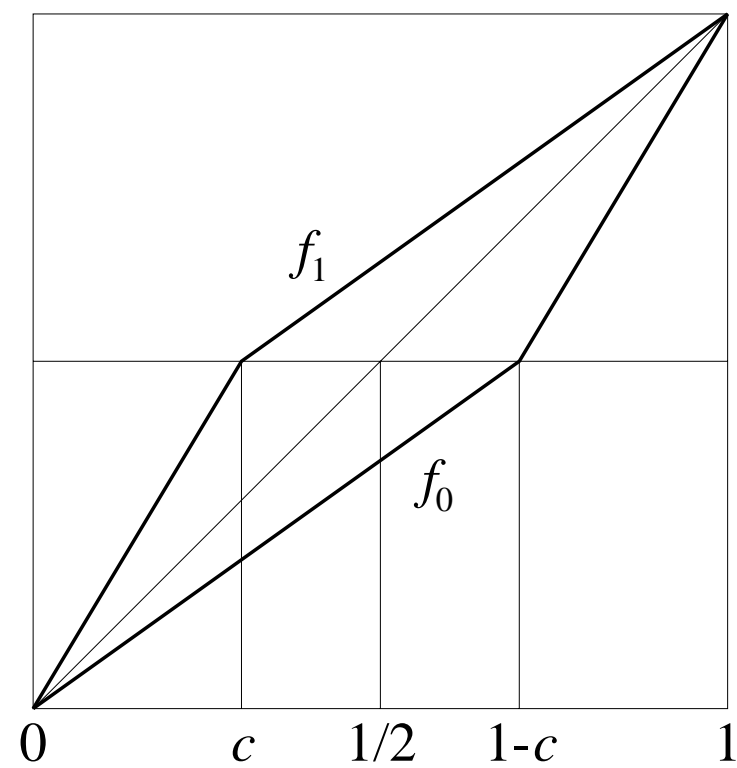

Figure 1. The maps $f_{0}$ and $f_{1}$

Then we use the metric $d(x, y)=|h(x)-h(y)|$. We can rewrite it as

$$
d(x, y)= \begin{cases}|\log (x)-\log (y)| & \text { if } x, y \in(0,1 / 2] \\ |\log (1-x)-\log (1-y)| & \text { if } x, y \in[1 / 2,1)\end{cases}
$$

and $d(x, y)=d(x, 1 / 2)+d(y, 1 / 2)$ in any other case. Clearly, $d$ is a metric in $(0,1)$, equivalent to the Euclidean one.

equaltriang Remark 5.1. If $x \leq y \leq z$ then $d(x, z)=d(z, y)+d(y, z)$.

Now we start the study of the contraction of $F$.

deriv Lemma 5.2. Assume that $1 / 2 \leq x<y<1$. Then

$$
\frac{\log y-\log x}{\log (1-x)-\log (1-y)} \leq \frac{4-2 y}{3} \text {. }
$$

Proof. We have

$$
\frac{\log y-\log x}{\log (1-x)-\log (1-y)}=\frac{\frac{\log y-\log x}{y-x}}{\frac{\log (1-x)-\log (1-y)}{(1-x)-(1-y)}} .
$$

Since the logarithmic function is concave, the numerator of the right-hand side above is a decreasing function of $x$, while the denominator is a decreasing function of $1-x$, that is, an increasing function of $x$. Therefore, the whole fraction is a decreasing function of $x$. Thus,

$$
\frac{\log y-\log x}{\log (1-x)-\log (1-y)} \leq \frac{\log y-\log \frac{1}{2}}{\log \frac{1}{2}-\log (1-y)}=\frac{\log 2 y}{-\log 2(1-y)}
$$

Assume that $0 \leq t<1$. We use two well-known estimates of the logarithm, namely

$$
\log (1+t) \leq t \quad \text { and } \quad-\log (1-t) \geq t+\frac{t^{2}}{2} .
$$


From those inequalities we get

$$
\frac{\log (1+t)}{-\log (1-t)} \leq \frac{t}{t+\frac{t^{2}}{2}}=\frac{2}{2+t}
$$

We claim that

$$
\frac{2}{2+t} \leq \frac{3-t}{3}
$$

Indeed, this is equivalent to $6 \leq 6-2 t+3 t-t^{2}$, that is, to $t(1-t) \geq 0$, which is true under our assumptions. From (5.3) and (5.4) we get

$$
\frac{\log (1+t)}{-\log (1-t)} \leq \frac{3-t}{3}
$$

Applying this inequality to $t=2 y-1$, we get

$$
\frac{\log 2 y}{-\log 2(1-y)} \leq \frac{4-2 y}{3} \text {. }
$$

Together with (5.2), we obtain (5.1).

f-contr Lemma 5.3. If either $x, y \in(0,1 / 2]$ or $x, y \in[1-c, 1)$ then $d\left(f_{0}(x), f_{0}(y)\right)=d(x, y)$. If $x, y \in[1 / 2,1-c]$ then

$$
d\left(f_{0}(x), f_{0}(y)\right) \leq\left(1-\frac{2 c}{3} d(x, y)\right) d(x, y) .
$$

If $x, y \in(0, c]$ or $x, y \in[1 / 2,1)$ then $d\left(f_{1}(x), f_{1}(y)\right)=d(x, y)$. If $x, y \in[c, 1 / 2]$ then (5.5) holds with $f_{1}$ instead of $f_{0}$.

Proof. We will only prove the statements for $f_{0}$. The statements for $f_{1}$ follow in a similar way (or one can use symmetry).

If $x, y \in(0,1 / 2]$, then

$$
d\left(f_{0}(x), f_{0}(y)\right)=|\log (a x)-\log (a y)|=|\log (x)-\log (y)|=d(x, y) .
$$

When $x, y \in[1-c, 1)$ we also obtain $d\left(f_{0}(x), f_{0}(y)\right)=d(x, y)$ in a similar way.

Now assume that $x, y \in[1 / 2,1-c]$ and $x<y$. Then

$$
d(x, y)=\log (1-x)-\log (1-y) \quad \text { and } \quad d\left(f_{0}(x), f_{0}(y)\right)=\log y-\log x .
$$

Thus, by Lemma 5.2,

$$
d\left(f_{0}(x), f_{0}(y)\right) \leq \frac{4-2 y}{3} d(x, y) .
$$

On the interval $[c, 1 / 2]$ the logarithmic function is Lipschitz continuous with the constant $1 / c$. Therefore

SO

$$
c d(x, y)=c(\log (1-x)-\log (1-y)) \leq y-x \leq y-\frac{1}{2},
$$

$$
\frac{4-2 y}{3}=1-\frac{2 y-1}{3}=1-\frac{2}{3}\left(y-\frac{1}{2}\right) \leq 1-\frac{2 c}{3} d(x, y) .
$$

From this and (5.6) we get (5.5). 
Fix $\underline{\omega} \in \Sigma$. For $x_{0} \in[0,1]$ we will write $x_{n}=\pi_{2}\left(F^{n}\left(\underline{\omega}, x_{0}\right)\right)$. Set

$$
\Gamma=\left\{\underline{\omega} \in \Sigma: \lim _{n \rightarrow \infty} \#\left\{k \in\{0,1, \ldots, n-1\}: \omega_{k}=0\right\}=\frac{1}{2}\right\} .
$$

By the Birkhoff Ergodic Theorem, $\mu(\Gamma)=1$.

In what follows, given $\underline{\omega} \in \Gamma$ and $x_{0} \in(0,1)$, for $n \geq 1$ we define $x_{n}:=f_{\omega_{n-1}}\left(x_{n-1}\right)$. Observe that $F^{n}\left(\underline{\omega}, x_{0}\right)=F\left(S^{n-1}(\underline{\omega}), x_{n-1}\right)=\left(S^{n}(\underline{\omega}), x_{n}\right)$.

InfVisits Lemma 5.4. Let $\underline{\omega} \in \Gamma$ and $x_{0} \in(0,1)$. Then there are infinitely many values of $n$ such that $x_{n} \in(0,1 / 2]$ and infinitely many values of $n$ such that $x_{n} \in[1 / 2,1)$.

Proof. Suppose that there are only finitely many $n$ 's such that $x_{n} \in[1 / 2,1)$. Without loss of generality we may assume that there are no such $n$ 's. Then $\omega_{n}=0$ implies $x_{n+1}=a x_{n}$ and $\omega_{n}=1$ implies $x_{n+1}=b x_{n}$. Take $\varepsilon>0$ such that

$$
\varepsilon<\frac{\log (a b)}{2 \log \left(\frac{b}{a}\right)} .
$$

Then, $a^{\frac{1}{2}+\varepsilon} b^{\frac{1}{2}-\varepsilon}>1$. Since $\underline{\omega} \in \Gamma$, if $n$ is large enough,

$$
\#\left\{k<n: \omega_{n}=0\right\}<\left(\frac{1}{2}+\varepsilon\right) n .
$$

Consequently,

$$
x_{n} \geq a^{\left(\frac{1}{2}+\varepsilon\right) n} b^{\left(\frac{1}{2}-\varepsilon\right) n} x_{0}=\left(a^{\frac{1}{2}+\varepsilon} b^{\frac{1}{2}-\varepsilon}\right)^{n} x_{0}
$$

and this last expression tends to $\infty$ as $n$ tends to $\infty$; a contradiction.

d-contr Lemma 5.5. For every $x, y \in(0,1)$ we have

$$
d\left(f_{0}(x), f_{0}(y)\right) \leq d(x, y) \quad \text { and } \quad d\left(f_{1}(x), f_{1}(y)\right) \leq d(x, y) .
$$

Proof. If both $x, y$ are in one of the intervals $(0,1 / 2]$ or $[1 / 2,1-c]$, or $[1-c, 1)$, then by Lemma $5.3 d\left(f_{0}(x), f_{0}(y)\right) \leq d(x, y)$. Otherwise, we divide the interval between $x$ and $y$ into two or three subintervals as above and use Remark 5.1.

For $f_{1}$ the proof is similar.

nohalf Lemma 5.6. There exists $\eta>0$ such that if $x \leq 1 / 2 \leq y$ and $d(x, y)<\eta$ then $f_{0}(x)<f_{0}(y)<1 / 2$ and $1 / 2<f_{1}(x)<f_{1}(y)$.

Proof. This follows immediately from the inequality $f_{0}(1 / 2)<1 / 2<f_{1}(1 / 2)$ and continuity of $f_{0}$ and $f_{1}$.

d-a-n-contr Lemma 5.7. Let $1 / 2 \leq x_{0}<y_{0}$ and $x_{n}<y_{n} \leq 1 / 2$ for some $n \geq 1$. Assume also that $d\left(x_{0}, y_{0}\right)<\eta$, where $\eta$ is the constant from the preceding lemma. Then

$$
d\left(x_{n}, y_{n}\right) \leq \frac{2+\frac{c}{3} d\left(x_{0}, y_{0}\right)}{2+\frac{2 c}{3} d\left(x_{0}, y_{0}\right)} d\left(x_{0}, y_{0}\right) .
$$

Proof. Let $k$ be the largest integer from $\{0,1, \ldots, n-1\}$ such that $1 / 2 \leq x_{k}<y_{k}$. By Lemmas 5.6 and 5.5, either $x_{k+1}=f_{0}\left(x_{k}\right)<y_{k+1}=f_{0}\left(x_{k}\right) \leq 1 / 2$ or $x_{k+2}=$ $f_{0}\left(x_{k+1}\right)<y_{k+2}=f_{0}\left(x_{k+1}\right) \leq 1 / 2$ (in the latter case, $\left.k \leq n-2\right)$. 
In the first case, by Lemma 5.3,

$$
d\left(x_{k+1}, y_{k+1}\right) \leq\left(1-\frac{2 c}{3} d\left(x_{k}, y_{k}\right)\right) d\left(x_{k}, y_{k}\right)
$$

so by Lemma 5.5,

$$
d\left(x_{n}, y_{n}\right) \leq\left(1-\frac{2 c}{3} d\left(x_{n}, y_{n}\right)\right) d\left(x_{0}, y_{0}\right)
$$

This inequality implies

$$
d\left(x_{n}, y_{n}\right) \leq \frac{1}{1+\frac{2 c}{3} d\left(x_{0}, y_{0}\right)} d\left(x_{0}, y_{0}\right) .
$$

If $\alpha>0$ then $1 /(1+2 \alpha)<(2+\alpha) /(2+2 \alpha)$, so (5.7) follows in this case.

In the second case there is a point $z_{0} \in\left(x_{0}, y_{0}\right)$ such that $z_{k+1}=1 / 2$. Then the first case applies if we replace $y_{0}$ by $z_{0}$, and also if we replace $x_{0}$ by $z_{0}$. Suppose that $d\left(x_{0}, z_{0}\right) \geq d\left(z_{0}, y_{0}\right)$ (if $d\left(x_{0}, z_{0}\right)<d\left(z_{0}, y_{0}\right)$ then the proof is similar). Then, by (5.8) (applied to $x_{0}$ and $z_{0}$ ), Lemma 5.5 and Remark 5.1, we get

$$
d\left(x_{n}, y_{n}\right)=d\left(x_{n}, z_{n}\right)+d\left(z_{n}, y_{n}\right) \leq \frac{1}{1+\frac{2 c}{3} d\left(x_{0}, z_{0}\right)} d\left(x_{0}, z_{0}\right)+d\left(z_{0}, y_{0}\right) .
$$

Since $d\left(x_{0}, z_{0}\right) \geq d\left(z_{0}, y_{0}\right)$ and $d\left(x_{0}, z_{0}\right)+d\left(z_{0}, y_{0}\right)=d\left(x_{0}, y_{0}\right)$, we have $d\left(x_{0}, z_{0}\right) \geq$ $d\left(x_{0}, y_{0}\right) / 2$, so we can write

$$
d\left(x_{0}, z_{0}\right)=d\left(x_{0}, y_{0}\right) / 2+\left(d\left(x_{0}, z_{0}\right)-d\left(x_{0}, y_{0}\right) / 2\right)
$$

with $d\left(x_{0}, z_{0}\right)-d\left(x_{0}, y_{0}\right) / 2 \geq 0$. Thus,

$$
\frac{1}{1+\frac{2 c}{3} d\left(x_{0}, z_{0}\right)} d\left(x_{0}, z_{0}\right) \leq \frac{1}{1+\frac{2 c}{3} d\left(x_{0}, z_{0}\right)} \cdot \frac{d\left(x_{0}, y_{0}\right)}{2}+\left(d\left(x_{0}, z_{0}\right)-\frac{d\left(x_{0}, y_{0}\right)}{2}\right) \text {. }
$$

Together with (5.9), taking into account that $d\left(x_{0}, z_{0}\right)+d\left(z_{0}, y_{0}\right)=d\left(x_{0}, y_{0}\right)$, we get

$$
d\left(x_{n}, y_{n}\right) \leq\left(\frac{1}{1+\frac{2 c}{3} d\left(x_{0}, z_{0}\right)}+1\right) \frac{d\left(x_{0}, y_{0}\right)}{2} .
$$

Using $d\left(x_{0}, z_{0}\right) \geq d\left(x_{0}, y_{0}\right) / 2$ again, we get

$$
d\left(x_{n}, y_{n}\right) \leq\left(\frac{1}{1+\frac{c}{3} d\left(x_{0}, y_{0}\right)}+1\right) \frac{d\left(x_{0}, y_{0}\right)}{2}=\frac{2+\frac{c}{3} d\left(x_{0}, y_{0}\right)}{2+\frac{2 c}{3} d\left(x_{0}, y_{0}\right)} d\left(x_{0}, y_{0}\right) .
$$

Thus, (5.7) also follows in this case.

Define a function $\chi:[0, \infty) \rightarrow \mathbb{R}$ by

$$
\chi(t)= \begin{cases}\frac{2+\frac{c}{3} t}{2+\frac{2 c}{3} t} t & \text { if } 0 \leq t \leq \frac{\eta}{2}, \\ \frac{2+\frac{c \eta}{6}}{2+\frac{c \eta}{3}} t & \text { if } t>\frac{\eta}{2},\end{cases}
$$

where $\eta$ is the constant from Lemma 5.6. It is easy to see that $\chi$ is continuous, $\chi(0)=0$ and $\chi(t)<t$ if $t>0$. Therefore, for every $t \geq 0$ we have

$$
\lim _{n \rightarrow \infty} \chi^{n}(t)=0 \text {. }
$$


It is clear that $\chi$ is strictly increasing on $[\eta / 2, \infty]$. By differentiating the first formula defining $\chi$, one can easily check that the same is true on $[0, \eta / 2]$. Thus, $\chi$ is invertible and for every $t>0$ we have

$$
\lim _{n \rightarrow \infty} \chi^{-n}(t)=\infty \text {. }
$$

Lemma 5.8. Let $1 / 2 \leq x_{0}<y_{0}$ and $x_{n}<y_{n} \leq 1 / 2$ for some $n \geq 1$. Then

$$
d\left(x_{n}, y_{n}\right) \leq \chi\left(d\left(x_{0}, y_{0}\right)\right) .
$$

Proof. If $d\left(x_{0}, y_{0}\right) \leq \eta / 2$, then (5.13) follows immediately from Lemma 5.7 and the definition of $\chi$. If $d\left(x_{0}, y_{0}\right)>\eta / 2$, then we can divide the interval $\left[x_{0}, y_{0}\right]$ by taking points $x_{0}=x_{0}^{0}<x_{0}^{1}<x_{0}^{2}<\ldots x_{0}^{m}=y_{0}$ such that $\eta / 2 \leq d\left(x_{0}^{i}, x_{0}^{i+1}\right)<\eta$ for $i=0,1, \ldots, m-1$, and apply Lemma 5.7 to each of the intervals $\left[x_{0}^{i}, x_{0}^{i+1}\right]$. We get

$$
d\left(x_{n}, y_{n}\right)=\sum_{i=0}^{m-1} d\left(x_{n}^{i}, x_{n}^{i+1}\right) \leq \sum_{i=0}^{m-1} \frac{2+\frac{c}{3} d\left(x_{0}^{i}, x_{0}^{i+1}\right)}{2+\frac{2 c}{3} d\left(x_{0}^{i}, x_{0}^{i+1}\right)} d\left(x_{0}^{i}, x_{0}^{i+1}\right) .
$$

Since for $t \geq \eta / 2$ we have

$$
\frac{2+\frac{c}{3} t}{2+\frac{2 c}{3} t} \leq \frac{2+\frac{c \eta}{6}}{2+\frac{c \eta}{3}}
$$

we obtain

$$
d\left(x_{n}, y_{n}\right) \leq \frac{2+\frac{c \eta}{6}}{2+\frac{c \eta}{3}} \sum_{i=0}^{m-1} d\left(x_{0}^{i}, x_{0}^{i+1}\right)=\frac{2+\frac{c \eta}{6}}{2+\frac{c \eta}{3}} d\left(x_{0}, y_{0}\right)=\chi\left(d\left(x_{0}, y_{0}\right)\right) .
$$

forw-contr Lemma 5.9. Let $\underline{\omega} \in \Gamma$ and let $x_{0}, y_{0} \in(0,1)$. Then $\lim _{n \rightarrow \infty} d\left(x_{n}, y_{n}\right)=0$.

Proof. We may assume that $x_{0}<y_{0}$. By Lemma 5.4, there are increasing sequences $\left(n_{k}\right)$ and $\left(m_{k}\right)$ such that $n_{k}<m_{k}<n_{k+1}$ and $y_{m_{k}} \leq 1 / 2 \leq x_{n_{k}}$. By Lemma 5.8 we have $d\left(x_{m_{k}}, y_{m_{k}}\right) \leq \chi\left(d\left(x_{n_{k}}, y_{n_{k}}\right)\right)$. By this and Lemma 5.5 used inductively, we get $d\left(x_{n_{k+1}}, y_{n_{k+1}}\right) \leq \chi\left(d\left(x_{n_{k}}, y_{n_{k}}\right)\right)$. Thus, by induction, $d\left(x_{n_{k}}, y_{n_{k}}\right) \leq \chi^{k-1}\left(d\left(x_{n_{1}}, y_{n_{1}}\right)\right)$. By (5.11), we get $\lim _{n_{k} \rightarrow \infty} d\left(x_{n_{k}}, y_{n_{k}}\right)=0$. Using again Lemma 5.5 inductively and taking into account that $n_{k}<n_{k+1}$ (so $n_{k} \rightarrow \infty$ as $k \rightarrow \infty$ ), we get $\lim _{n \rightarrow \infty} d\left(x_{n}, y_{n}\right)=$ 0 .

The derivative of the function $h$, which is used to define distance $d$, is larger than 1 . Therefore $|x-y| \leq d(x, y)$ for all $x, y \in(0,1)$. In such a way we get from Lemma 5.9 the desired result.

forw-contr1 Theorem 5.10. For almost all $\underline{\omega} \in \Sigma$, if $x_{0}, y_{0} \in(0,1)$ then $\lim _{n \rightarrow \infty}\left|x_{n}-y_{n}\right|=0$.

exmain Corollary 5.11. The map F considered in this section satisfies the assumptions of Theorem 3.2.

1-sid-contr Remark 5.12. If instead of $F$ we consider the map $F_{+}$, which is a skew product over the one-sided shift, for a given $\underline{\omega} \in \Sigma_{+}$and $x_{0} \in I$ we get the same $x_{n}$ as for $F$ when we replace $\underline{\omega}$ by any two-sided sequence with the same $\omega_{k}$ for $k \geq 0$. Therefore Theorem 5.10 holds also if we replace $\Sigma$ by $\Sigma_{+}$and $F$ by $F_{+}$. 


\section{Measures}

We continue to investigate $F$ and $F_{+}$, this time from the point of view of invariant measures. The relevant invariant measures for $F$ and $F_{+}$are those that project to $\mu$ and $\mu_{+}$. There are two trivial ergodic ones: $\mu \times \delta_{0}$ and $\mu \times \delta_{1}$ (in the one-sided case, $\mu_{+} \times \delta_{0}$ and $\left.\mu_{+} \times \delta_{1}\right)$.

By Theorem 3.3 and Corollary 5.11, there is at most one nontrivial measure of this type. Such measure for $F$ is $\mu_{\varphi}$, which appears in Theorem 3.2 (e). It is clear that the projection from $\Sigma \times I$ to the first coordinate is an isomorphism of the systems $\left(\Sigma \times I, F, \mu_{\varphi}\right)$ and $(\Sigma, \sigma, \mu)$. In particular, this shows that $\mu_{\varphi}$ is ergodic for $F$.

Now we consider $F_{+}$. Here the situation is completely different. Denote the Lebesgue measure on $I$ by $\lambda$. The following theorem can be interpreted as the Lebesgue measure being invariant for our random system of maps. The proof is straightforward and specific for our family.

invmeas Theorem 6.1. The measure $\mu_{+} \times \lambda$ is invariant for $F_{+}$.

Proof. Let $\varepsilon_{i} \in\{0,1\}$ for $i=0,1, \ldots, n-1$ and let

$$
C=C\left(\varepsilon_{0}, \varepsilon_{1}, \ldots, \varepsilon_{n-1}\right):=\left\{\left(\omega_{0}, \omega_{1}, \ldots\right): \omega_{i}=\varepsilon_{i} \text { for } i=0,1, \ldots, n-1\right\}
$$

be an $n$-cylinder of the one-sided shift and let $A \subset[0,1 / 2]$ or $A \subset[1 / 2,1]$ be a $\lambda$-measurable set.

Then, $F_{+}^{-1}(C \times A)=\left(C_{0} \times A_{0}\right) \cup\left(C_{0} \times A_{1}\right)$, where, for $j \in\{0,1\}, A_{j}=f_{j}^{-1}(A)$ and

$$
C_{j}=\left\{\underline{\omega}: \omega_{0}=j \text { and } \omega_{i}=\varepsilon_{i} \text { for } i=1,2, \ldots, n-1\right\} .
$$

Since $\frac{1}{a}+\frac{1}{b}=2$ we have $\lambda\left(A_{0}\right)+\lambda\left(A_{1}\right)=\frac{\lambda(A)}{a}+\frac{\lambda(A)}{b}=2 \lambda(A)$ and clearly $\mu_{+}\left(C_{j}\right)=$ $\frac{1}{2} \mu_{+}(C)$. Therefore,

$$
\left(\mu_{+} \times \lambda\right)\left(F_{+}^{-1}(C \times A)\right)=\frac{1}{2} \mu_{+}(C) \cdot 2 \lambda(A)=\left(\mu_{+} \times \lambda\right)(C \times A) .
$$

The sets of the form $C \times A$ with $C, A$ as above generate the whole $\sigma$-field of $\mu_{+} \times \lambda$-measurable sets. This completes the proof.

Once we know this measure, let us compute the Lyapunov exponent in the direction of the fiber. For each $f_{j}$, the derivative is $a$ on an interval of length $1 /(2 a)$ and $b$ on an interval of length $1 /(2 b)$. Therefore the exponent is

$$
\frac{1}{2 a} \log a+\frac{1}{2 b} \log b \text {. }
$$

We have $1 /(2 a)=1-c$ and $1 /(2 b)=c$. Therefore

$$
\begin{aligned}
\frac{1}{2 a} \log a+\frac{1}{2 b} \log b & =(1-c)(-\log 2-\log (1-c))+c(-\log 2-\log c) \\
& =-(1-c) \log (1-c)-c \log c-\log 2 .
\end{aligned}
$$

Since $0<c<1 / 2$, this exponent is negative. This agrees with Theorem 5.10.

Recall that $P: \Sigma \rightarrow \Sigma_{+}$is the natural projection (that forgets about $\omega_{n}$ with negative $n$ ).

image_meas Proposition 6.2. We have

$$
\left(P \times \operatorname{id}_{I}\right)_{*}\left(\mu_{\varphi}\right)=\mu_{+} \times \lambda .
$$


Proof. We have $P_{*}(\mu)=\mu_{+}$, so $\left(P \times \operatorname{id}_{I}\right)_{*}\left(\mu_{\varphi}\right)$ is a measure invariant for $F_{+}$. This measure vanishes on the set $\Sigma_{+} \times\{0,1\}$, so by Theorems 3.3 and 6.1 it is equal to $\mu_{+} \times \lambda$.

Let us comment on invariant measures for the random systems we are considering. We assume that $F_{+}$is essentially contracting and the base system is Bernoulli. By Theorem 3.3, there is one nontrivial measure invariant for $F_{+}$that projects to $\mu_{+}$. By Theorem 4.2, it is of the form $\mu_{+} \times \nu$ for some measure $\nu$ on the interval. Thus, the question about the existence of an absolutely continuous measure for our system is the question whether this specific measure $\nu$ is absolutely continuous. This is very different from the situation for non-random interval maps, when there is a lot of invariant measures and we are asking only whether there is one among them which is absolutely continuous. We conjecture that typically (whatever this means) the measure $\nu$ is not absolutely continuous. The systems considered in Theorem 6.1 are very special, and $\nu=\lambda$ just follows from the definition of the maps.

Now we can prove some interesting properties of the function $\varphi$.

dense Theorem 6.3. For almost every $x \in I$ the preimage $\varphi^{-1}(x)$ is dense in $\Sigma$. In particular, the graph of $\varphi$ is dense in $\Sigma \times I$.

Proof. Choose a cylinder $C=C\left(\varepsilon_{-n}, \varepsilon_{-n+1}, \ldots, \varepsilon_{n}\right)$, analogous as in the proof of Theorem 6.1. By Lemma 4.1, on $\sigma^{n}(C)$ the function $\varphi$ takes all values that it takes on the whole space. However, by Proposition 6.2 and since $\mu_{\varphi}$ is concentrated on the graph of $\varphi$, it takes almost all values from $I$. By Remark 2.3,

$$
\varphi\left(\sigma^{n}(\underline{\omega})\right)=\pi_{2}\left(F^{n}(\underline{\omega}, \varphi(\underline{\omega}))\right) .
$$

If $\underline{\omega} \in \mathbb{C}$, then

$$
\pi_{2}\left(F^{n}(\underline{\omega}, \varphi(\underline{\omega}))\right)=\left(f_{\varepsilon_{-n+1}} \circ f_{\varepsilon_{-n+2}} \circ \cdots \circ f_{\varepsilon_{0}}\right)(\varphi(\underline{\omega})) .
$$

The map $f_{\varepsilon_{-n+1}} \circ f_{\varepsilon_{-n+2}} \circ \cdots \circ f_{\varepsilon_{0}}$ is a homeomorphism preserving the Lebesgue equivalence class, and therefore $\varphi$ takes on $C$ almost all values from $I$.

Cylinders form a countable basis of the topological space $\Sigma$ and the intersection of a countable family of sets of full measure has full measure. Therefore for almost every $x \in I$ the preimage $\varphi^{-1}(x)$ is dense in $\Sigma$.

The second statement of the theorem follows immediately from the first one.

\section{TWO-SIDED VS. ONE SIDED CASE}

By Theorem 3.2 (c) and Corollary 5.11, the map $F$ has a fiberwise attractor which is a graph of a measurable invariant function from the base to the fiber space. We will show that this is not the case if we consider $F_{+}$, even if we skip the assumption of invariance.

noattr Theorem 7.1. There is no measurable function $\varphi_{+}: \Sigma_{+} \rightarrow(0,1)$ whose graph is an attractor for $F_{+}$in the sense that for almost every $\underline{\omega} \in \Sigma_{+}$and every $x_{0} \in(0,1)$ we have

$$
\lim _{n \rightarrow \infty}\left|x_{n}-\varphi_{+}\left(\sigma_{+}^{n}(\underline{\omega})\right)\right|=0 .
$$


Proof. Assume that such $\varphi_{+}$exists. Then the graph of $\varphi_{+} \circ P: \Sigma \rightarrow(0,1)$ is an attractor for $F$, because $x_{n}$ depends only on $x_{0}$ and on $\omega_{k}$ with nonnegative $k$. By a theorem from [1], $\varphi_{+} \circ P=\varphi$ almost everywhere. Thus, the graph of $\varphi_{+} \circ P$ is $F$-invariant, and it follows that the graph of $\varphi_{+}$is $F_{+}$-invariant.

The measure $\left(\mu_{+}\right)_{\varphi_{+}}$is then a nontrivial $F_{+}$-invariant ergodic measure, so by Theorems 3.3 and 6.1 it is equal to $\mu_{+} \times \lambda$, a contradiction.

In such a way we get an excellent illustration of the Mystery of the Vanishing Attractor, described in [1]. For an invertible system an attractor exists, but it vanishes when we pass to the noninvertible system. This happens in spite of the fact that in the definition of an attractor we only look at forward orbits, and that in the base the future is completely independent of the past.

One can try to explain this paradox by saying that for $F_{+}$also there is an attractor, but it is the whole space. This is true, but normally when thinking of an attractor one considers subsets much smaller than the whole space. Another explanation is that when trying to find an attractor for $F_{+}$, which is a graph, we try to specify one point in $(0,1)$ for each $\underline{\omega} \in \Sigma_{+}$, without specifying $x_{0}$. However, when we know the past, we basically know $x_{0}$, and with the knowledge of $x_{0}$ and $\underline{\omega} \in \Sigma_{+}$we know $x_{n}$ for all $n \geq 0$. Again, this is a kind of explanation (due to M. Rams), but still the question why in order to have a nice description of the future we need the past, if the past and the future are independent, remains a little mysterious.

\section{REFERENCES}

[1] Ll. Alsedà and M. Misiurewicz, Skew Product Attractors and concavity, preprint, 2012.

[2] V. Bergelson, M. Misiurewicz and S. Senti, Affine actions of a free semigroup on the real line, Ergod. Th. Dynam. Sys. 26 (2006), 1285-1305.

BoMi [3] A. Bonifant and J. Milnor, Schwarzian derivatives and cylinder maps, in "Holomorphic dynamics and renormalization", Fields Inst. Commun. 53, Amer. Math. Soc., Providence, RI, 2008, pp. $1-21$.

[4] W. de Melo and S. van Strien, "One-Dimensional Dynamics", Springer Verlag, Berlin, 1983.

[5] M. Misiurewicz, Absolutely continuous measures for certain maps of an interval, Publ. Math. IHES 53 (1981), 17-51.

Departament de Matemàtiques, Edifici Cc, Universitat Autònoma de Barcelona, 08913 Cerdanyola del Vallès, Barcelona, Spain

E-mail address: alseda@mat.uab.cat

Department of Mathematical Sciences, IUPUi, 402 N. Blackford Street, IndiANAPOLIS, IN 46202

E-mail address: mmisiure@math.iupui.edu 